\title{
Hydromagnetic Instability of Two Viscoelastic Dusty-Fluids in Porous Medium
}

\author{
PARDEEP KUMAR*1, SUMIT GUPTA ${ }^{2}$ \\ ${ }^{1}$ Department of Mathematics, ICDEOL, Himachal Pradesh University, Summerhill, \\ Shimla-5, INDIA \\ ${ }^{2}$ Department of Mathematics, Govt. Degree College Arki (Solan) HP, INDIA.
}

\begin{abstract}
An attempt has been made to investigate the instability of the plane interface between two viscoelastic superposed conducting fluids in the presence of suspended particles and variable horizontal magnetic field through porous medium is studied. The cases of two fluids of uniform densities, viscosities, magnetic fields, and suspended particles number densities separated by a horizontal boundary; and of exponentially varying density, viscosity, suspended particles number density, and magnetic field are considered. It is found that the stability criterion is independent of the effects of viscoelasticity, medium porosity, and suspended particles but is dependent on the orientation and magnitude of the magnetic field. The magnetic field succeeds in stabilizing a certain range of wavenumbers which were unstable in the absence of the magnetic field. The system is found to be stable for potentially stable configuration/stratification. The growth rates are found to increase (for certain wavenumbers) and decrease (for other wavenumbers) with the increase in kinematic viscosity, suspended particles number density, magnetic field, medium permeability and stress relaxation time.
\end{abstract}

Key-words: Maxwellian viscoelastic fluids, porous medium, Rayleigh-Taylor instability, suspended particles, variable horizontal magnetic field,

Received: April 9, 2021. Revised: August 22, 2021. Accepted: September 2, 2021. Published: September 20, 2021.

\section{Introduction}

When two fluids of different densities are superposed one over the other (or accelerated towards each other), the instability of the plane interface between the two fluids, when it occurs, is called RayleighTaylor instability. In general, it is derived from the character of the equilibrium of an incompressible stratified heterogeneous fluid. Mention may be made of two important special cases: (a) two fluids of different densities superposed one over the other; (b) a fluid with continuous density stratification. Hide [1] has treated the character of the equilibrium of a viscous, incompressible, rotating fluid of variable density and found that rotation stabilizes the potentially unstable arrangement for certain wavenumber range. He has carried the directions of angular velocity vector and gravity vector (in the direction of the vertical) to be inclined. In another study, Hide [2] has studied the case of a viscous, incompressible, electrically conducting fluid of variable density in the presence of a vertical magnetic field and found that magnetic field considerably stabilizes the configuration and it is possible to have oscillatory motions in the presence of magnetic field even if the configuration is thoroughly unstable (density wise). Chandrasekhar [3] has given a detailed account of the instability of the plane interface between two incompressible and viscous fluids of different densities when the lighter is accelerated into the heavier. The influence of viscosity on the stability of the plane interface separating two electrically conducting, incompressible superposed fluids of uniform densities, when the whole system is immersed in a uniform horizontal magnetic field, has been studied by Bhatia [4]. He has carried out the stability analysis for two fluids of high viscosities and different uniform densities. A good account of stability problems has also been given by Joseph and Renardy [5] in their study of two-fluid dynamics. Hoshoudy and El-Ansary [6] have studied the effect of viscosity 
and homogeneous horizontal magnetic field on Rayleigh-Taylor instability of a heavy fluid supported by a lighter one.

In geophysical situations, more often than not, the fluid is not pure but may instead be permeated with suspended (or dust) particles. The effect of suspended particles on the stability of superposed fluids might be of industrial and chemical engineering importance. Further, motivation for this study is the fact that knowledge concerning fluidparticle mixtures is not commensurate with their industrial and scientific importance. Scanlon and Segel [7] have considered the effect of suspended particles on the onset of Be'nard convection and found that the critical Rayleigh number was reduced solely because the heat capacity of the pure gas was supplemented by that of the particles. The effect of suspended particles was thus found to destabilize the layer. Palaniswamy and Purushotham [8] have studied the stability of shear flow of stratified fluids with fine dust and found the effect of fine dust to increase the region of instability. Alloui et al. [9] have studied the onset of double-diffusive convection in a horizontal Brinkman cavity and analysis made on the linear stability of the quiescent state within a horizontal porous cavity subject to vertical gradients of temperature and solute. Recently spacecraft observations have confirmed that the dust particles play an important role in the dynamics of atmosphere as well as in the diurnal and surface variations in the temperature of the Martian weather. It is, therefore, of interest to study the presence of suspended particles in astrophysical situations. The medium has been considered to be non-porous in all the above studies.

The flow through porous media is of considerable interest for petroleum engineers, for geophysical fluid dynamicists and has importance in chemical technology and industry. An example in the geophysical context is the recovery of crude oil from the pores of reservoir rocks. Among the applications in engineering disciplines one can find the food processing industry, chemical processing industry, solidification and centrifugal casting of metals. Such flows has shown their great importance in petroleum engineering to study the movement of natural gas, oil and water through the oil reservoirs; in chemical engineering for filtration and purification processes and in the field of agriculture engineering to study the underground water resources, seepage of water in river beds. The problem of Rayleigh-Taylor instability in a porous medium is of importance in geophysics, soil sciences, ground water hydrology and astrophysics. The study of Rayleigh-Taylor instability of fluids saturated porous media has diverse practical applications, including that related to the materials processing technology, in particular, the melting and solidification of binary alloys. The development of geothermal power resources has increased general interest in the properties of convection in porous media. The scientific importance of the field has also increase because hydrothermal circulation is the dominant heattransfer mechanism in young oceanic crust (Lister, [10]). Generally it is accepted that comets consists of a dusty 'snowball' of a mixture of frozen gases which in the process of their journey changes from solid to gas and vice-versa. The physical properties of comets, meteorites and interplanetary dust strongly suggest the importance of porosity in the astrophysical context (McDonnel, [11]).

Many common materials such as paints, polymer's, plastics and more exotic one such as silicic magma, saturated soils and the Earth's lithosphere behaves as viscoelastic fluids. With the growing importance of non-Newtonian fluids in geophysical fluid dynamics, chemical technology and petroleum industry, the investigations on such fluids are desirable. The stability of a horizontal layer of Maxwell's viscoelastic fluid heated from below has been investigated by Vest and Arpaci [12]. The nature of instability and some factors may have different effects on viscoelastic fluids as compared to the Newtonian fluids. For example, Bhatia and Steiner [13] have considered the effect of a uniform rotation on the thermal instability of a Maxwell fluid and have found that rotation has a destabilizing effect in contrast to the stabilizing effect on Newtonian fluid. Bhatia and Steiner [14] have also considered the thermal instability of a Maxwell fluid in hydromagnetics and have found that the magnetic field has a stabilizing effect on viscoelastic fluid as in the case of Newtonian fluid. Kumar and Singh [15] studied the superposed Maxwellian viscoelastic fluids through porous media in hydromagnetics. Kumar et al. [16] also studied the instability of two rotating Maxwellian viscoelastic superposed fluids with variable magnetic field in porous medium. Kumar and Mohan [17] considered the thermosolutal convection in a heterogeneous Maxwellian fluid layer heated and soluted from below through porous medium. The triply diffusive convection in a Maxwell viscoelastic fluid in the presence of uniform vertical magnetic field through porous medium has been considered by Sharma et al. [18]. Kumar [19] has studied the transport of vorticity in a magnetic 
Maxwellian viscoelastic fluid in the presence of suspended magnetic particles.

Keeping in mind the importance in chemical technology, biomechanics, paper and pulp technology, oil recovery and various importance stated above of porous media, suspended particles and magnetic field, the present paper attempts to study the effects of suspended particles and variable horizontal magnetic field on the Rayleigh-Taylor instability of Maxwellian viscoelastic fluids in porous medium.

\section{Formulation of the Problem and Perturbation Equations}

Let $\quad \Gamma_{i j}, \tau_{i j}, e_{i j}, \delta_{i j}, p, u_{i}, x_{i}, \mu$ and $\lambda \quad$ denote, respectively, the stress tensor, shear stress tensor, rate of strain tensor, Kronecker delta, scalar pressure, velocity, position vector, viscosity, and stress relaxation time. Then the Maxwellian viscoelastic fluid is described by the constitutive relations

$$
\left.\begin{array}{c}
\Gamma_{i j}=-p \delta_{i j}+\tau_{i j} \\
\left(1+\lambda \frac{d}{d t}\right) \tau_{i j}=2 \mu e_{i j} \\
e_{i j}=\frac{1}{2}\left(\frac{\partial u_{i}}{\partial x_{j}}+\frac{\partial u_{j}}{\partial x_{i}}\right)
\end{array}\right\},
$$

where

$$
\frac{d}{d t}=\frac{\partial}{\partial t}+u_{j} \frac{\partial}{\partial x_{j}}
$$

is the "convective derivative".

Consider a static state in which an incompressible and infinitely (electrically) conducting Maxwellian viscoelastic fluid permeated with suspended particles is arranged in horizontal strata in porous medium and the pressure $p$ and density $\rho$ are functions of the vertical coordinate $z$ only. The system is acted on by gravity force $\vec{g}(0,0,-g)$ and variable horizontal magnetic field $\vec{H}(H(z), 0,0)$. The character of the equilibrium of this initial static state is determined, as usual, by supposing that the system is slightly disturbed and then by following its further evolution.

Let $\vec{u}(u, v, w), \vec{h}\left(h_{x}, h_{y}, h_{z}\right), \delta \rho$, and $\delta p$ denote, respectively, the perturbations in fluid velocity $(0,0,0)$, magnetic field $\vec{H}$, density $\rho$, and pressure $p ; \vec{v}(\bar{x}, t)$ and $N(\bar{x}, t)$ denote the velocity and the number density of the particles respectively. $K=$
$6 \pi \rho v \eta$, where $\eta$ is the particle radius, is the Stokes drag coefficient, $\vec{v}=(l, r, s)$ and $\bar{x}=(x, y, z)$. Then the linearized perturbation equations of Maxwellian viscoelastic fluid through porous medium in the presence of variable magnetic field, using (1) and Darcy's law, are

$$
\begin{aligned}
\frac{\rho}{\varepsilon}\left(1+\lambda \frac{\partial}{\partial t}\right) \frac{\partial \vec{u}}{\partial t} & =\left(1+\lambda \frac{\partial}{\partial t}\right)[-\nabla \delta p+g \delta \rho \\
& +\frac{\mu_{e}}{4 \pi}\{(\nabla \times \vec{h}) \times \vec{H}+(\nabla \times \vec{H}) \times \vec{h}\} \\
& \left.+\frac{K N}{\varepsilon}(\vec{v}-\vec{u})\right] \\
& -\frac{\mu}{k_{1}} \vec{u}
\end{aligned}
$$

$\nabla \cdot \vec{u}=0$,

$$
\varepsilon \frac{\partial \vec{h}}{\partial t}=\nabla \times(\vec{u} \times \vec{H})
$$

$$
\nabla \cdot \vec{h}=0 \text {, }
$$

$$
\varepsilon \frac{\partial}{\partial t} \delta \rho=-w \frac{d \rho}{d z} .
$$

Here $\mu, \varepsilon, k_{1}, \mu_{e}, N$, and $g$ stand for fluid viscosity, medium porosity, medium permeability, magnetic permeability, number density of particles, and acceleration due to gravity, respectively. Assuming uniform particles size, spherical shape, and small relative velocities between the fluid and particles, the presence of particles adds an extra force term in the equations of motion (2), proportional to the velocity difference between particles and fluid.

Since the force exerted by the fluid on the particles is equal and opposite to that exerted by the particles on the fluid, there must be an extra force term, equal in magnitude but opposite in sign, in the equations of motion for the particles. The buoyancy force on the particles is neglected. Interparticle reactions are also ignored, for we assume that distances between the particles are quite large compared with their diameters. If $m N$ is the mass of particles per unit volume, the linearized perturbed equations of motion and continuity for the particles, under the above assumptions are

$$
\begin{aligned}
& \left(\frac{m}{K} \frac{\partial}{\partial t}+1\right) \vec{v}=\vec{u}, \\
& \frac{\partial M}{\partial t}+\nabla \cdot \vec{v}=0,
\end{aligned}
$$

where $M=\varepsilon N / N_{0}$; and $N_{0}, N$ stand for initial uniform number density and perturbation in number density, respectively. Equation (6) ensures that the 
density of each particle remains unchanged as we follow it with its motion.

Analyzing the disturbances into normal modes, we seek solutions whose dependence on $x, y$ and $t$ is given by

$$
\exp \left(i k_{x} x+i k_{y} y+n t\right)
$$

where $k_{x}, k_{y}$ are horizontal wave numbers; $k^{2}=$ $k_{x}^{2}+k_{y}^{2}$; and $n$ is a complex constant.

For perturbation of the form (9), equations (2) - (7), after eliminating $\vec{v}$, give

$$
\begin{gathered}
(1+\lambda n)\left[\frac{\rho}{\varepsilon}(\tau n+1)+\frac{m N}{\varepsilon}\right] n u+(1+\tau n) \frac{\mu}{k_{1}} u \\
=(1+\lambda n)(1+\tau n)\left[-i k_{x} \delta p\right. \\
\left.+\frac{\mu_{e}}{4 \pi} h_{z}(D H)\right], \\
(1+\lambda n)\left[\frac{\rho}{\varepsilon}(\tau n+1)+\frac{m N}{\varepsilon}\right] n v+(1+\tau n) \frac{\mu}{k_{1}} v \\
=(1+\lambda n)(1+\tau n)\left[-i k_{y} \delta p\right. \\
\left.+\frac{\mu_{e}}{4 \pi} H\left(i k_{x} h_{y}-i k_{y} h_{x}\right)\right],(11) \\
(1+\lambda n)\left[\frac{\rho}{\varepsilon}(\tau n+1)+\frac{m N}{\varepsilon}\right] n w+(1+\tau n) \frac{\mu}{k_{1}} w= \\
(1+\lambda n)(1+\tau n)[-D \delta p \\
+\frac{\mu_{e}}{4 \pi} H\left(i k_{x} h_{z}-D h_{x}-h_{x} \frac{D H}{H}\right) \\
-g \delta \rho], \quad \\
i k_{x} u+i k_{y} v+D w=0, \\
\varepsilon n h_{x}=i k_{x} H u-w(D H), \\
\varepsilon n h_{y}=i k_{x} H v, \\
\varepsilon n h_{z}=i k_{x} H w, \\
i k_{x} h_{x}+i k_{y} h_{y}+D h_{z}=0, \\
\varepsilon n(\delta \rho)=-w(D \rho),
\end{gathered}
$$

where $\tau=m / K$, and $D=d / d z$.

Multiplying equations (10) and (11) by $-i k_{x},-i k_{y}$, respectively, and adding, using equations $(13)-(18)$ in it, and finally eliminating $\delta p$ between the resulting equation and equation (12), we obtain

$$
\begin{gathered}
(1+\lambda n)(1+\tau n) \frac{n}{\varepsilon}\left[D(\rho D w)-k^{2} \rho w\right] \\
+(1+\lambda n) \frac{n}{\varepsilon}[D(m N D w) \\
\left.-k^{2} m N w\right]+\frac{(1+\tau n)}{k_{1}} \\
{\left[D(\mu D w)-\mu k^{2} w\right]+\frac{g k^{2}}{\varepsilon n}(1+\lambda n)} \\
(1+\tau n)(D \rho) w-(1+\lambda n) \\
(1+\tau n) \frac{\mu_{e} k_{x}^{2}}{4 \pi \varepsilon n}\left[k^{2} H^{2} w-D\left(H^{2} D w\right)\right] \\
=0
\end{gathered}
$$

\section{Special Cases}

\section{Case I: Two Uniform Maxwellian Viscoelastic Fluids Separated by a Horizontal Boundary}

Consider the case of two uniform Maxwellian viscoelastic fluids of densities $\rho_{1}$ and $\rho_{2}$, viscosities $\mu_{1}$ and $\mu_{2}$, uniform suspended particles densities $N_{1}$ and $N_{2}$, and uniform magnetic fields $H_{1}$ and $H_{2}$, separated by a horizontal boundary at $z=0$. The subscripts 1 and 2 distinguishes the lower and upper fluids, respectively. Then in each region of constant , constant $\mu$, constant $m N$, and constant $H$, equation (19) reduces to

$$
\left(D^{2}-k^{2}\right) w=0 .
$$

Since $w$ must vanish both when $z \rightarrow \infty$ (in the upper fluids), and $z \rightarrow-\infty$ (in the lower fluid), the general solution of equation (20) can be written as

$$
\begin{aligned}
& w_{1}=A e^{k z}(z<0), \\
& w_{2}=A e^{-k z}(z>0),
\end{aligned}
$$

where the same constant $A$ has been chosen to ensure the continuity of $w$ at $z=0$.

Integrating equation (19) across the interface $\quad z=$ 0 , we obtain the boundary condition

$$
\begin{gathered}
(1+\lambda n)(1+\tau n) \frac{n}{\varepsilon}\left[\rho_{2} D w_{2}-\rho_{1} D w_{1}\right]_{z=0} \\
+(1+\lambda n) \frac{m n}{\varepsilon}
\end{gathered}
$$




$$
\begin{aligned}
{\left[N_{2} D w_{2}-N_{1} D\right.} & \left.w_{1}\right]_{z=0} \\
& +\frac{(1+\tau n)}{k_{1}}\left[\mu_{2} D w_{2}-\mu_{1} D w_{1}\right]_{z=0} \\
& +\frac{g k^{2}}{\varepsilon n}(1+\lambda n)(1+\tau n)\left(\rho_{2}\right. \\
& \left.-\rho_{1}\right) w_{0}+(1+\lambda n)(1+\tau n)
\end{aligned}
$$$$
\frac{k_{x}^{2} V_{A}^{2}}{\varepsilon n}\left[\rho_{2} D w_{2}-\rho_{1} D w_{1}\right]_{z=0}=0 \text {, }
$$

where $w_{0}$ is the common value of $w$ at $z=0$. For the sake of simplicity, we have considered that the Alfv'en velocities of the two fluids are the same, so that

$$
V_{A}^{2}=\frac{\mu_{e} H_{1}^{2}}{4 \pi \rho_{1}}=\frac{\mu_{e} H_{2}^{2}}{4 \pi \rho_{2}} .
$$

Applying the condition (23) to the solutions (21) and (22), we obtain

$$
\begin{aligned}
{\left[\lambda \tau\left(\rho_{2}+\rho_{1}\right)\right] n^{4} } & \\
& +\left[(\lambda+\tau)\left(\rho_{2}+\rho_{1}\right)\right. \\
& \left.+m \lambda\left(N_{1}+N_{2}\right)\right] n^{3} \\
& +\left[\left(\rho_{2}+\rho_{1}\right)+m\left(N_{1}+N_{2}\right)\right. \\
& +\frac{\varepsilon \tau\left(\mu_{1}+\mu_{2}\right)}{k_{1}} \\
& +\lambda \tau\left\{-g k\left(\rho_{2}-\rho_{1}\right)\right. \\
& \left.\left.+k_{x}^{2} V_{A}^{2}\left(\rho_{2}+\rho_{1}\right)\right\}\right] n^{2} \\
& +\left[\frac{\varepsilon}{k_{1}}\left(\mu_{2}+\mu_{1}\right)\right. \\
& +(\lambda+\tau)\left\{-g k\left(\rho_{2}-\rho_{1}\right)\right. \\
& \left.\left.+k_{x}^{2} V_{A}^{2}\left(\rho_{2}+\rho_{1}\right)\right\}\right] n \\
& +\left[-g k\left(\rho_{2}-\rho_{1}\right)\right. \\
& \left.+k_{x}^{2} V_{A}^{2}\left(\rho_{2}+\rho_{1}\right)\right]=0 .(24)
\end{aligned}
$$

Theorem 1: For the potentially stable configuration, the system is always stable.

Proof: For the potentially stable configuration $\left(\rho_{2}<\rho_{1}\right)$, all the coefficients of equation (24) are positive, and so all the roots of the equation (24) are either real and negative, or there are complex roots (which occur in pairs) with negative real parts. The system is therefore stable in each case. The potentially stable configuration, therefore, remains stable whether the fluid is viscoelastic (Maxwellian) and permeated with suspended particles in porous medium in hydromagnetics or not.

Theorem 2: For the unstable configuration, the system is stable or unstable according as $k_{x}^{2} V_{A}^{2}$ is greater than or smaller than $g k\left(\alpha_{2}-\alpha_{1}\right)$ and the magnetic field succeeds in stabilizing a certain range of wavenumbers.

Proof: For the potentially unstable configuration $\left(\rho_{2}>\rho_{1}\right)$, if

$$
k_{x}^{2} V_{A}^{2}\left(\rho_{2}+\rho_{1}\right)>g k\left(\rho_{2}-\rho_{1}\right),
$$

that is, if

$$
k_{x}^{2} V_{A}^{2}>g k\left(\alpha_{2}-\alpha_{1}\right),
$$

where

$$
\alpha_{1,2}=\frac{\rho_{1,2}}{\rho_{1}+\rho_{2}} .
$$

Equation (24) does not admit any change of sign and has no positive root. Therefore, the system is stable.

However, if

$$
k_{x}^{2} V_{A}^{2}<g k\left(\alpha_{2}-\alpha_{1}\right),
$$

the constant term in equation (24) is negative, so there is at least one change of sign in equation (24). Equation (24) allows at least one positive root of $n$, meaning thereby instability of the system. The stability criterion (25) is independent of the effects of viscoelasticity, medium porosity, and suspended particles.

Thus for the unstable case, the system is stable or unstable according as $k_{x}^{2} V_{A}^{2}$ is greater than or smaller than $g k\left(\alpha_{2}-\alpha_{1}\right)$. The magnetic field thus stabilizes a certain wavenumber range $k>k^{*}$, where

$$
k^{*}=\frac{g\left(\alpha_{2}-\alpha_{1}\right)}{V_{A}^{2}} \sec ^{2} \theta,
$$

for the unstable configuration even in the presence of suspended particles, and porous medium effects. The critical wavenumber $k^{*}$, above which the system is stabilized, is dependent on the magnitude of the magnetic field and fluid densities as well as the orientation $\theta$ of the magnetic field. Here $\theta$ is the angle between $\vec{k}$ and $\vec{H}$; that is, $k_{x}=k \cos \theta$.

\section{Case II: Case of Exponentially Varying Stratifications}

Assume the stratifications in fluid density, fluid viscosity, suspended particles number density, and magnetic field of the form

$$
\begin{gathered}
\rho=\rho_{0} e^{\beta z}, \mu=\mu_{0} e^{\beta z}, N=N_{0} e^{\beta z}, H^{2} \\
=H_{0}^{2} e^{\beta z},
\end{gathered}
$$

where $\rho_{0}, \mu_{0}, N_{0}, H_{0}$, and $\beta$ are constants. Equations (29) imply that: 
Kinematic viscosity $v\left(=\frac{\mu}{\rho}=\frac{\mu_{0}}{\rho_{0}}=v_{0}\right), \frac{N}{\rho}\left(=\frac{N_{0}}{\rho_{0}}\right)$, and Alfve'n velocity $V_{A}\left(=\sqrt{\left(\frac{\mu_{e} H^{2}}{4 \pi \rho}\right)}=\sqrt{\left(\frac{\mu_{e} H_{0}^{2}}{4 \pi \rho_{0}}\right)}\right)$ are constant everywhere.

Substituting (29) in equation (19), and neglecting the effect of heterogeneity on inertia, we obtain

$$
\left(D^{2}-k^{2}\right) w+\frac{g \beta k^{2} / n^{2}}{\left[1+\frac{h}{\tau n+1}+\frac{k_{\chi}^{2} V_{A}^{2}}{n^{2}}+\frac{v_{0}}{k_{1}(1+\lambda n) \frac{n}{\varepsilon}}\right]} w
$$$$
=0 \text {, }
$$

where $h=m N_{0} / \rho_{0}$.

Consider the case of two free boundaries which although somewhat artificial except for stellar atmosphere, where it is the most appropriate (Spiegel [20]), provides analytical solutions. The boundary conditions in this case are

$$
w=D^{2} w=0 \quad \text { at } z=0 \text { and } z=d .
$$

The appropriate solution of (30) satisfying (31) is

$$
w=A \sin \left(\frac{m \pi z}{d}\right),
$$

where $m$ is an integer.

Substituting (32) in (30), we obtain the dispersion relation

$$
\begin{aligned}
& n^{4}+\frac{1}{\lambda \tau}[(\lambda+\tau)+\lambda h] n^{3} \\
& +\frac{1}{\lambda \tau}\left[1+h+\frac{\varepsilon v_{0}}{k_{1}} \tau+\left(k_{x}^{2} V_{A}^{2}-\frac{g \beta k^{2}}{L}\right) \lambda \tau\right] n^{2} \\
& +\frac{1}{\lambda \tau}\left[\frac{\varepsilon v_{0}}{k_{1}}+(\lambda+\tau)\left\{k_{x}^{2} V_{A}^{2}-\frac{g \beta k^{2}}{L}\right\}\right] n \\
& +\frac{1}{\lambda \tau}\left[k_{x}^{2} V_{A}^{2}-\frac{g \beta k^{2}}{L}\right] \\
& =0
\end{aligned}
$$

where $L=\frac{m^{2} \pi^{2}}{d^{2}}+k^{2}$.

Theorem 3: System is stable for stable stratifications.

Proof: For $\beta<0$ (stable stratifications), equation (33) does not allow any positive root of $n$ and so the system is stable.

Theorem 4: System is stable or unstable for unstable stratifications. Magnetic field succeeds in stabilizing a certain range of wavenumbers which were unstable in the absence of the magnetic field.

Proof: For $\beta>0$ (unstable stratifications), the system is stable or unstable according as

$$
k_{x}^{2} V_{A}^{2} \gtrless \frac{g \beta k^{2}}{L} .
$$

In the absence of magnetic field, the system is unstable for $\beta>0$. However, the system can be completely stabilized by a large magnetic field, as can be seen from equation (33), if

$$
V_{A}^{2}>\frac{g \beta k^{2}}{k_{x}^{2} L} .
$$

The magnetic field, therefore, succeeds in stabilizing a wavenumber range

$$
k^{2}>\frac{g \beta}{V_{A}^{2}} \sec ^{2} \theta-\frac{m^{2} \pi^{2}}{d^{2}},
$$

in which the wavenumbers were unstable in the absence of a magnetic field. The viscoelasticity, suspended particles, and medium porosity do not have any qualitative effect on the nature of stability or instability.

Theorem 5: The growth rates increase (for certain wavenumbers) and decrease (for other wavenumbers) with the increase in kinematic viscosity, suspended particles number density, magnetic field. Medium permeability and stress relaxation time.

Proof: If

$$
\beta>0 \text { and } k_{x}^{2} V_{A}^{2}<\frac{g \beta k^{2}}{L},
$$

the equation (33) has one positive root. Let $n_{0}$ denote the positive root of equation (33). Then

$$
\begin{aligned}
n_{0}^{4}+\frac{1}{\lambda \tau}[(\lambda+\tau) & +\lambda h] n_{0}^{3} \\
& +\frac{1}{\lambda \tau}\left[1+h+\frac{\varepsilon v_{0}}{k_{1}} \tau\right. \\
& \left.+\left(k_{x}^{2} V_{A}^{2}-\frac{g \beta k^{2}}{L}\right) \lambda \tau\right] n_{0}^{2} \\
& +\frac{1}{\lambda \tau}\left[\frac{\varepsilon v_{0}}{k_{1}}\right. \\
& \left.+(\lambda+\tau)\left\{k_{x}^{2} V_{A}^{2}-\frac{g \beta k^{2}}{L}\right\}\right] n_{0} \\
& +\frac{1}{\lambda \tau}\left[k_{x}^{2} V_{A}^{2}-\frac{g \beta k^{2}}{L}\right] \\
& =0
\end{aligned}
$$


To find the role of viscosity, magnetic field, suspended particles number density, medium permeability, and stress relaxation time on the growth rate of unstable modes, we examine the nature of

$d n_{0} / d v_{0}, d n_{0} / d V_{A}, d n_{0} / d h, d n_{0} / d k_{1}, \quad$ and $d n_{0} / d \lambda$. Equation (37) yields

$$
\frac{d n_{0}}{d v_{0}}=-\frac{\varepsilon n_{0}}{E k_{1} \lambda \tau}\left(1+\tau n_{0}\right),
$$

where

$$
\begin{aligned}
E=4 n_{0}^{3}+\frac{3}{\lambda \tau}[ & (\lambda+\tau)+\lambda h] n_{0}^{2} \\
& +\frac{2}{\lambda \tau}\left[1+h+\frac{\varepsilon v_{0}}{k_{1}} \tau\right. \\
& \left.+\left(k_{x}^{2} V_{A}^{2}-\frac{g \beta k^{2}}{L}\right) \lambda \tau\right] n_{o} \\
& +\frac{1}{\lambda \tau}\left[\frac{\varepsilon v_{0}}{k_{1}}\right. \\
& +(\lambda+\tau)\left\{k_{x}^{2} V_{A}^{2}\right. \\
& \left.\left.-\frac{g \beta k^{2}}{L}\right\}\right] .
\end{aligned}
$$

It is evident from (38) and (39) that $d n_{0} / d v_{0}$ may be positive or negative. A similar result holds for $d n_{0} / d V_{A}, d n_{0} / d h, d n_{0} / d k_{1}$, and $d n_{0} / d \lambda$.

The growth rates, therefore, both decrease (for certain wavenumbers) and increase (for other wavenumbers) with the increase in kinematic viscosity, magnetic field, suspended particles number density, medium permeability, and stress relaxation time.

\section{Conclusions}

A detailed account of stability of superposed Newtonian fluids under varying assumptions of hydrodynamics and hydromagnetics, was given by Chandrasekhar [3]. The Rayleigh-Taylor instability of Maxwellian fluids in the presence of suspended particles and variable horizontal magnetic field in a porous medium is considered in the present paper. The motivation for the present study is due to the fact that many common materials such as paints, polymers, plastics, and more exotic ones such as silicic magma, saturated soils, and the Earth's lithosphere, behave as viscoelastic fluids, and the importance of a variable magnetic field, suspended particles and porous medium in chemical engineering and geophysics. The main results of the present study are:

(i) The dispersion relation studying the effect of variable horizontal magnetic field, suspended particles, viscosity, viscoelasticity on the stability of fluids separated by horizontal boundary and stratified (exponentially varying density, viscosity, suspended particles number density, magnetic field) fluid in porous medium has been obtained.

For stable configuration, the system is found to be stable whereas for the unstable case, the system is stable or unstable according as $k_{x}^{2} V_{A}^{2}$ is greater than or less than $g k\left(\alpha_{2}-\alpha_{1}\right)$.

For stable stratification, the system is found to be stable for disturbances of all wave-numbers.

For unstable stratification, the system is stable or unstable according as $k_{x}^{2} V_{A}^{2}$ is greater than or less than $\left(g \beta k^{2} / L\right)$ '

The magnetic field stabilizes unstable stratification for small wave-length perturbations $\quad k^{2}>\frac{g \beta}{V_{A}^{2}} \sec ^{2} \theta-\frac{m^{2} \pi^{2}}{d^{2}}$ which were instable in its absence.

(vi) The stability criterion is independent of the effects of viscoelasticity, medium porosity, and suspended particles but is dependent on the orientation and magnitude of the magnetic field.

(vii) It is also found that the growth rates increase (for certain wavenumbers) and decrease (for other wavenumbers) with the increase in kinematic viscosity, suspended particles number density, magnetic field, medium permeability, and stress relaxation time.

\section{Acknowledgements}

The authors are grateful to all the three learned referees for their useful technical comments and valuable suggestions, which led to a significant improvement of the paper. 


\section{References}

[1] Hide, R., Waves in a heavy, viscous, incompressible electrically conducting fluid of variable density in the presence of a magnetic field, Proc. Roy. Soc. Lond., Vol. A233, 1955, pp. 376379.

[2] Hide, R., The character of the equilibrium of a heavy, viscous, incompressible rotating fluid of variable density: I. General theory, Q. J. Mech. Appl. Math., Vol. 9, 1956, pp. 22-34.

[3] Chandrasekhar, S., Hydrodynamic and Hydromagnetic Stability, Dover Publication, New York, 1981.

[4] Bhatia, P.K., Rayleigh-Taylor instability of two viscous superposed conducting fluids, Nuovo Cimento, Vol. B19, 1974, pp. 161-168.

[5] Joseph, D.D. and Renardy, Y.Y., Fundamentals of Two-Fluid Dynamics, Part I, Math. Theory and Application, Springer, New York, 1993.

[6] Hoshoudy, G.A. and El-Ansary, N.F., Study of the effect of viscosity and homogeneous horizontal magnetic field on Rayleigh-Taylor instability, $Z$. Angew. Math. Mech., Vol. 83 (1), 2003, pp. 61-68.

[7] Scanlon, J.W. and Segel, L.A., Some effects of suspended particles on the onset of Be'nard convection, Phys. Fluids, Vol. 16, 1973, pp. 15731578 .

[8] Palaniswamy, V.I. and Purushotham, C.M., Stability of shear flow of stratified fluids with fine dust, Phys. Fluids, Vol. 24, 1981, pp. 1224-1229.

[9] Alloui, Z., Vasseur, P., Robillard, L. and Bahloul, A., Onset of double-diffusive convection in a horizontal Brinkman cavity, Che. Engng. Comm., Vol. 197, 2010, pp. 387-399.

[10] Lister, P.F., On the thermal balance of a midocean ridge, Geophys. J. Royal Astro. Soc., Vol. 26, 1972, pp. 515-535.

[11] McDonnel, J.A.M., Cosmic Dust, Toronto, Canada, John Wiley \& Sons, 1978.

[12] Vest, C.M. and Arpaci, V.S., Overstability of a viscoelastic fluid layer heated from below, J. Fluid Mech., Vol. 36, 1969, pp. 613-619.

[13] Bhatia, P.K. and Steiner, J.M., Convective instability in a rotating viscoelastic fluid layer, $Z$. Angew Math. Mech., Vol. 52, 1972, pp. 321-327.
[14] Bhatia, P.K. and Steiner, J.M., Thermal instability in a viscoelastic fluid layer in hydromagnetics, J. Math. Anal. Appl., Vol. 41, 1973, pp. 271-283.

[15] Kumar, P. and Singh, M., On superposed Maxwellian viscoelastic fluids through porous medium in hydromagnetics, Int. e-Journal Engng. Math.: Theory and Appl., Vol. 3(1), 2008, pp. 146158.

[16] Kumar, P., Singh, M. and Singh, G.J., Instability of two rotating Maxwellian viscoelastic superposed fluids with variable magnetic field in porous medium, Acta Ciencia Indica (India), Vol. XXXIV M(3), 2008, pp. 1449-1458.

[17] Kumar, P. and Mohan, H., Rayleigh-Taylor instability through porous medium of two viscoelastic (Maxwellian) superposed fluids, Int. J. Appl. Sci. Math., Vol. 1(2), 2014, pp. 68-73.

[18] Sharma, P.K., Malik, H., Kumar, V. and Kumar, P., Triply-diffusive magneto convection in viscoelastic fluid through porous medium, Int. Trans. Appl. Sci., Vol. 6(4), 2014, pp. 495-510.

[19] Kumar, P., Vorticity transport in magnetic Maxwellian viscoelastic fluid, Int. J. Math. Anal. Appl., Vol. 4(5), 2017, pp. 26-30.

[20] Spiegel, E.A., Convective instability in a compressible atmosphere, Astrophys. J., Vol. 141, 1965, pp. 1068-1090.

\section{Creative Commons Attribution License 4.0} (Attribution 4.0 International, CC BY 4.0) This article is published under the terms of the Creative Commons Attribution License 4.0 https://creativecommons.org/licenses/by/4.0/deed.en $\underline{\text { US }}$ 